\title{
Evaluation of a System for Automatic Detection of Diabetic Retinopathy from Color Fundus Photographs for Screening Population
}

\author{
Raju S. Maher \\ Department of Computer \\ Science \& IT \\ Dr. Babasaheb Ambedkar \\ Marathwada University, \\ Aurangabad.
}

\author{
Sangramsing N. Kayte \\ Department of Computer \\ Science \& IT \\ Dr. Babasaheb Ambedkar \\ Marathwada University, \\ Aurangabad.
}

\author{
Mukta Dhopeshwarkar \\ Department of Computer \\ Science \& IT \\ Dr. Babasaheb Ambedkar \\ Marathwada University, \\ Aurangabad.
}

\begin{abstract}
Diabetic Retinopathy is an eye disease and a common complication of diabetes that can cause vision loss if left undiagnosed at the initial stage. It is the prime cause of blindness in the working age population of the world. Colour fundus images of eyes are used by ophthalmologists to study eye diseases like diabetic retinopathy. Detection method is proposed to detect dark or red lesions such as microaneurysms and hemorrhages in fundus images. This method comprises of three stages. First, the green channel of the colour retinal image is preprocessed using polynomial contrast enhancement. Second, the candidate dark lesion objects are extracted from the contrast enhanced fundus image. A novel method is developed to extract candidate dark lesions based on matched filtering and local relative entropy thresholding. The performance of this technique is compared to mathematical morphology based dark lesion detection method. A hybrid method that combines both detection schemes is also tested for better segmentation. The final stage classifies true dark lesions from dark non-lesions using knn and svm classifiers. For image based evaluation, this method has resulted with sensitivity, specificity and accuracy of $94.86 \%$ and $95.6 \% 95.38 \%$ respectively.
\end{abstract}

\section{Keywords}

Biomedical image processing, retinal Fundus images, microaneurysm detection, morphological operator.

\section{INTRODUCTION}

We Eye is a very essential and critical organ of the human body which only gives vision. There are huge eye diseases increasing now days due to improper care. Diabetic Retinopathy (DR) is severe and wide diffusion diseases. It has been identified as one of the cause for blindness or vision impairment[1]. Diabetic retinopathy (DR) is a vascular disease of the retina which affects patients with the diabetes. It is the number one cause of blindness in people between the ages of 20-64 in the United States. Early DR detection and timely treatment have been shown to prevent visual loss and blindness in patients with retinal difficulties of diabetes[2]. In the next decade, projections for the United States are that the average age will rise, the number of people with diabetes in each age category will increases, and there will be a restriction of capable eye care providers [5]. The healthcare trends will challenge the public health capacity to care for both patients with DR and people with diabetes at risk for this difficulty. If the previous situation plays out, it will be necessary to either screen large numbers of people with diabetes for DR, ration access to eye care, or both.

The automated algorithms introduced above are improved to recommend recommendation for a patient with any form of DR to an ophthalmologist, and they were enhanced to detect early DR, because in our view, this is the burden[3]. They could be modified to diagnose vision threatening DR, detect those patients with important non proliferative DR, or proliferative DR[4]. This previews but not newly evaluated early detection programs. In addition, the processes were limited to detection of that called microaneurysms and small hemorrhages, to make the comparison though we have previously designed and evaluated systems that also detect exudates and cotton-wool spots[7]. The automated algorithms are thus optimized to recommend transfer for a patient with any form of DR to an ophthalmologist [6].

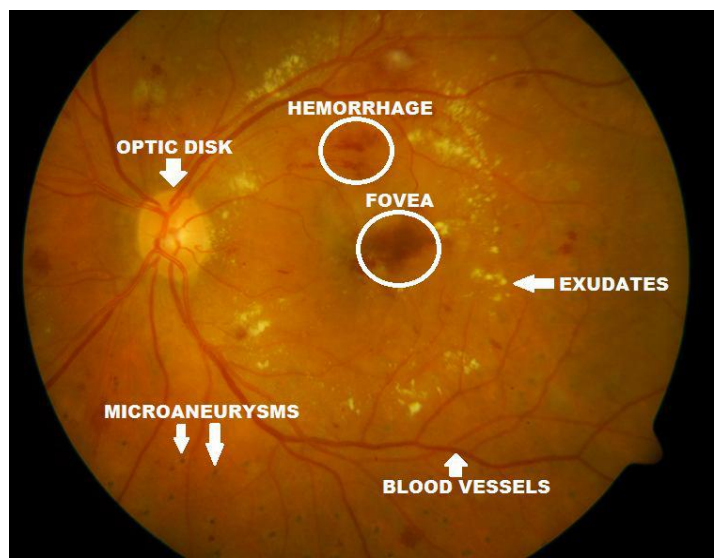

Fig. 1: Anatomical structures of fundus image.

The retina is a thin multi-layered sensory tissue that covers the inside wall at the back side part of eye is called fundus. It is covered number of. These photo receptors are responsible for receiving light rays, converting them into electrical impulses and then transmitting these signal to the brain where they are convert into images. Figure 1 shows a typical normal retinal fundus image with highlighted regions optic disc, fovea, blood macula and vessels. The outlying parts of the retina are responsible for outlying vision while the central area, that is called macula, is in charge of central vision that allows us to see details and perform tasks that require central vision, like was a reading. The circular area in the central region is a macula and that the retina measuring about $5 \mathrm{~mm}$ to $6 \mathrm{~mm}$ in diameter. A small gloom in the center of macula measuring about $1.5 \mathrm{~mm}$ in diameter is that is called fovea. 
The fovea corresponds to the highest sensitivity region of retina.

\subsection{Diabetic Retinopathy}

Diabetic retinopathy, the most major causes of common diabetic eye disease blindness is occurs when in the retina blood vessels change. Sometimes these vessels swell and leak fluid or block. In some cases, abnormal new blood vessels grow on the surface of the retina its last stage of DR. It is classified into two category first is Non-Proliferative Diabetic Retinopathy (NPDR) and Proliferative Diabetic Retinopathy (PDR).

\subsection{Non proliferative diabetic retinopathy}

Non proliferative diabetic retinopathy is the most common and may arise at the point in time after the diabetes. Ophthalmologists detect these changes by examining the patient's retina and look for spots of bleeding or swelling areas of retinal. Identification and recording of the following abnormalities (will aid in the accurate assessment of retinopathy severity.

\section{Microaneurysms}

The primary abnormality that occurs in the eye due to DR is the Microaneurysm. These are identified as tiny, dark red spots or miniscule haemorrhages, either appearing alone or in clusters, inherent to the light sensitive retina. The Microaneurysm is circular in shape and the size varies from 10-100 microns i.e. 1/12th the diameter of an average optics disc. The disease is not alarming at this phase.

\section{Hemorrhages}

These are also termed 'blot' microaneurysm, with concern to their round shape. These are found in the deeper layers of the retina. Intra retinal hemorrhages appear when capillaries or microaneurysms rupture and some blood leaks out of these vessels. In Figure 1.3 hemorrhages can be seen as red flame shaped regions.

\section{Hard Exudates}

Hard exudates represent leak of fluid that is rich in fat and protein from surrounding capillaries and microaneurysms within the retina. That is main features of diabetic retinopathy and appear as random yellowish patches of changing sizes, and shapes.

\section{Soft Exudates}

These are often called cotton wool spots and often seen in advanced retinopathy. These abnormalities usually appear as little round in the retina with a yellowish colour, usually adjacent to an area of hemorrhage. Cotton wool spots come to the swelling of the surface layer of the retina in the nonappearance of normal blood flow through the retinal blood vessels. The nerve fibers are injured and that resulting in swelling and appearance of a cotton wool spot [2].

\subsection{Proliferative diabetic retinopathy}

Proliferative diabetic retinopathy is the advanced stage retinopathy develops in more than 50 percent cases after about 25 years of onset of the disease. Therefore, it is more common in patients [9]. The hallmark of PDR is the growth of new blood vessels in the areas where normal capillaries have already closed. These new blood vessels are abnormal and fragile. They grow along the retina and along the surface of the clear, vitreous gel that fills the inside of the eye. By themselves, these blood vessels do not cause symptoms or vision loss. However, they have thin, fragile walls that leak blood resulting in severe vision loss and even blindness can be the end result.

\section{METHODS}

Fundus imaging has a main role in diabetic retinopathy monitoring since incidences of retinal abnormalities are common and consequences serious. However, since the eye fundus seems to be sensitive to vascular diseases, fundus imaging is considered as a candidate for non-invasive screening of diabetes. The details of these databases are forming the ground-truth of each image, which is also available in the DIARETDB0. However, one problem presented in this ground-truth is the low accuracy in the marking of lesions, since ophthalmologists delimited areas with a set of lesions and not each lesion itself. In this new ground-truth, among the 130 images, 109 images were defined with lesions and 21 images were defined no lesions. The technique for automatic detection of microaneurysms and hemorrhages proposed in this paper is based on mathematical morphology four stages. At the first stage, a preprocessing of the fundus images is performed in order to improve the contrast of these images. At the second stage, an improvement of the low intensity structures (blood vessels, fovea and red lesions) is performed. The blood vessels are detected by the sum of morphological operation using linear structuring elements with different angles in third stage. At the fourth stage, occurs the deduction of the blood vessels from the image obtained at the second stage. Each stage is explained in details in the following sections.

\subsection{Pre-processing}

Pre-processing is to reduce this effect by enhancing the contrast and normalizing the mean intensity. The objective of preprocessing is to attenuate the noise, to improve the contrast and to correct the non-uniform illumination. The colour retinal images taken at screening programs are often poorly contrasted and contain artifacts. To correct uneven illumination of images, a morphological top-hat operator with disk-shaped structuring element and fixed radius of 25 pixels was applied to the green component of the colour image. To reduce noise, a $3 \times 3$ median filter is applied to the shade corrected image [4].

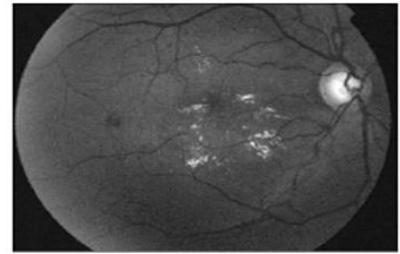

(a)

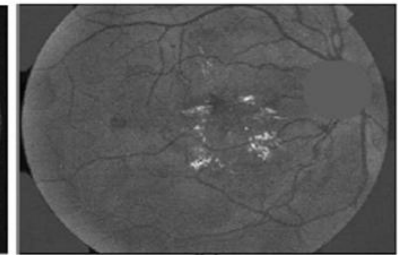

(b)
Fig. 2 - An image before and after the pre-processing operations, (a) green channel image, (b) pre-processed image.

\subsection{Microaneurysm Detection}

The extraction of microaneurysms step results in a set of candidate microaneurysm objects. The objective of the microaneurysm candidate classification system is to classify the objects as either microaneurysms or dark non-lesions. Hence to discard dark non-lesion candidates, classifiers are used which are trained with the features derived from the candidates. The best classification requires good features as well as good classifier.

$$
G_{2}=\psi^{\varsigma_{1}}\left(G_{1}\right)
$$


The image resulting from the local variation operator is denoted by G3 as below:

$$
G_{3}(x)=\frac{1}{N-1} \sum_{i \in w(x)}\left(G_{2}(i)-\mu(x)\right)^{2}
$$

where $\mathrm{x}$ is a set of all pixels in a sub-window $\mathrm{w}(\mathrm{x}), \mathrm{N}$ is the number of pixels in $w(x), \mu(x)$ is the mean value of G2(i) and $\mathrm{i}_{-} \mathrm{w}(\mathrm{x})$. The selection of window size is relied on the preferred compromise between the sensitivity and precision performance measures. So as the window size is larger the small exudates are more difficult to be detected leading to low sensitivity. Based on the experimental tests, we found that a window size of $9 \times 9$ gives good results.

\section{RESULTS AND DISCUSSION}

The proposed detection methods are tested and evaluated on DIARETDB0 [13], a publicly available database of colored fundus images and corresponding ground truth images. The entire process of segmenting vessels was performed on Intel PC with $1.66 \mathrm{GHz} \mathrm{CPU}$ and 512MB memory using Matlab 2012. The processing of each image including difficulty and thresholding took about30 seconds. The green component, of the RGB fundus image, was chosen to obtain the microaneurysms. Similar to the exudates detection algorithm, first the prominent structures within retina images, Such as blood vessel tree and optic disc are to be removed. After that a sophisticated sequence of image processing algorithms was used to determine the areas within the fundus images to get microaneurysms [14] and exudates. It is important to have some balance between the values of sensitivity and specificity. parameters such as True Positive (TP), True Negative (TN), False Positive (FP) and False Negative (FN). TP is the number of abnormal images classified as abnormal by the screening system. TN is the number of images that are really normal and classified as normal by the screening procedure. FP is the number of normal images that are predicted to be abnormal and FN is the number of abnormal images, classified by the procedure to be normal. Using these parameters, Sensitivity, Specificity and accuracy are calculated. For each of the images a corresponding manually segmented image is provided. The instruction of an ophthalmologist are coloured white. Quantitative evaluation of the segmentation algorithm is done by comparing the output image with the corresponding manually segmented image. In the classification stage, a training segmented dark non-lesion objects and dark lesion Table 1. Performance Comparison of Mathematical Morphology Detection. False positives are pixels marked as vessel by the method, but that are actually negatives in the ground truth. True negatives are pixels marked as background in both images The performance of the method was tested on images with incidence of lesions provided by the DIARETDB1. The accounting of the pixels in TP, FN, FP and TN was performed for each resulting image of the method. Table 1 shows the average values of TP, FN, FP and TN for each fundus image.

Table 1. Result value

\begin{tabular}{|c|c|c|c|c|c|}
\hline $\begin{array}{c}\text { Databas } \\
\text { e }\end{array}$ & $\begin{array}{c}\text { Total } \\
\text { imag } \\
\text { es }\end{array}$ & $\begin{array}{c}\text { Non- } \\
\text { exudat } \\
\text { es }\end{array}$ & $\begin{array}{c}\text { No. of } \\
\text { images } \\
\text { with }\end{array}$ & $\begin{array}{c}\text { Sensi } \\
\text { tivity } \\
(\%)\end{array}$ & $\begin{array}{c}\text { Speci } \\
\text { ficity } \\
(\%)\end{array}$ \\
\hline $\begin{array}{c}\text { Diaretdb } \\
0\end{array}$ & 130 & 58 & 72 & $\begin{array}{c}96.9 \\
\%\end{array}$ & $96.1 \%$ \\
\hline
\end{tabular}

False positives are pixels marked as vessel by the method, but that are actually negatives in the ground truth. True negatives are pixels marked as background in both images The performance of the method was tested on images with incidence of lesions provided by the DIARETDB1. The accounting of the pixels in TP, FN, FP and TN was performed for each resulting image of the method. Table 1 shows the average values of TP, FN, FP and TN for each fundus image.

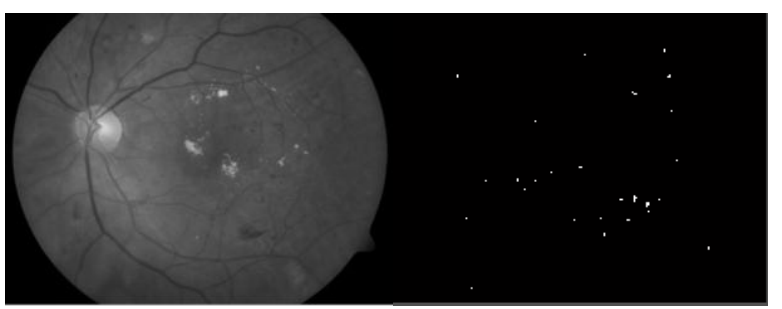

Fig. 3(a) shows the green channel of a fundus image Fig. 3(b) shows the detected microaneurysms

Lesion based evaluation and image based evaluation are employed to measure the accuracy of the proposed detection method at the pixel level. The recommend that any process used for screening sight threatening diabetic retinopathy should have at least $96.9 \%$ sensitivity and $96.1 \%$ specificity; Sensitivity means the percentage of abnormal fundus classified as abnormal by the procedure. Specificity means the percentage of normal funduses classified as normal by the procedure. The higher the sensitivity and specificity values, best the procedure.
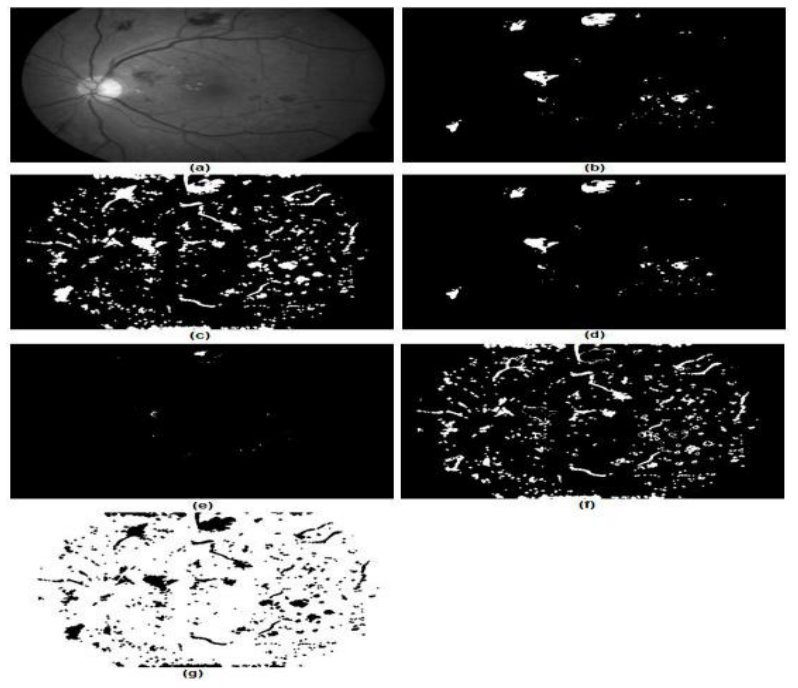

Figure 4. (a) Green channel of the fundus image. (b)

Ground truth image. (c) Microaneurysms and hemorrhages detected. (d) True positive pixels. (e) False negative pixels. (f) False positive pixels. (g) True negative pixels.

Table 2 Comparison of Performance with other technique

\begin{tabular}{|l|l|l|l|l|}
\hline $\begin{array}{l}\text { Autho } \\
\text { rs }\end{array}$ & Method & $\begin{array}{l}\text { Accuracy } \\
\text { of } \\
\text { classifica } \\
\text { tion }\end{array}$ & $\begin{array}{l}\text { Sensitivi } \\
\text { ty }\end{array}$ & $\begin{array}{l}\text { Specif } \\
\text { icity }\end{array}$ \\
\hline $\begin{array}{l}\text { Wang } \\
\text { et al. } \\
\text { 2000[5 } \\
\text { ] }\end{array}$ & $\begin{array}{l}\text { Minimum } \\
\text { distance } \\
\text { discriminant } \\
\text { classifier }\end{array}$ & $70 \%$ & $83 \%$ & $89 \%$ \\
\hline
\end{tabular}




\begin{tabular}{|l|l|c|c|c|}
\hline $\begin{array}{l}\text { Vujose } \\
\text { vic et } \\
\text { al.2009 } \\
\text { [6] }\end{array}$ & $\begin{array}{l}\text { Single } \\
\text { lesions }\end{array}$ & $\begin{array}{c}\text { Not } \\
\text { reported }\end{array}$ & $82 \%$ & $92 \%$ \\
\hline $\begin{array}{l}\text { Achary } \\
\text { a et al. } \\
\text { 2009[7 } \\
]\end{array}$ & $\begin{array}{l}\text { Blood } \\
\text { vessel, } \\
\text { exudates, } \\
\text { microaneury } \\
\text { sms, }\end{array}$ & $86 \%$ & $82 \%$ & $86 \%$ \\
\hline $\begin{array}{l}\text { Propo } \\
\text { sed } \\
\begin{array}{l}\text { Metho } \\
\text { d }\end{array}\end{array}$ & $\begin{array}{l}\text { Support } \\
\text { Vector } \\
\text { Machine } \\
\text { classifier }\end{array}$ & $\mathbf{9 5 . 3 8 \%}$ & $\mathbf{9 5 . 6 \%}$ & $\begin{array}{c}\mathbf{9 4 . 8 6} \\
\%\end{array}$ \\
\hline
\end{tabular}

The proposed method achieved satisfactory results the red lesions that were detected. These structures are mainly caused by remains of blood vessels, where there was ineptitude of the method to eliminate some vessels without eliminating lesions close to them. One factor that affected the sensitivity was not classifying some microaneurysms occupant area very small. Some of these lesions were confused with noise and were improperly removed due to its very small size, it may also be mentioned that the un-label brightness of some images generated false positive results, which reduced the specificity of the method.

\section{CONCLUSION}

The proposed method is adapted to deal with different types and qualities of images through taking all image information into account. The proposed method performance of the measured against clinician hand-labelled images. Comparing with some recent related works, the proposed method indicates an improvement in the specificity and accuracy measures and reasonable sensitivity particularly with the high performance method in. The performance results, demonstrated in this work, indicate that automated processing methods that are based on split-and-merge algorithm can give very competitive results in exudate detection. The strength of the proposed method comes from using computed parameters based on analysis of the image. A limitation in our work is that it occasionally fails to exclude some non-exudate objects particularly those that have similar features to real exudates.

\section{REFERENCES}

[1] 1. Raju Maher, Dr.Mukta Dhopeshwarkar "Automated Detection of Non-proliferative Diabetes Retinopathy Using Fundus Images" International Journal of Advanced Research in Computer Science and Software Engineering Volume 5, Issue 3, March 2015.

[2] Bresnick GH, Mukamel DB, Dickinson JC, Cole DR. A screening approach to the surveillance of patients with diabetes for the presence of vision-threatening retinopathy. Ophthalmology. 2000;107:19-24.

[3] Kinyoun JL, Martin DC, Fujimoto WY, Leonetti DL. Ophthalmoscopy versus fundus photographs for detecting and grading diabetic retinopathy. Invest Ophthalmol Vis Sci. 1992;33:1888-93.

[4] Raju Sahebrao Maher, Sangramsing N. Kayte, Sandip T. Meldhe, Mukta Dhopeshwarkar, "Automated Diagnosis Non-proliferative Diabetic Retinopathy in Fundus Images using Support Vector Machine" International Journal of Computer Applications (0975 - 8887)Volume 125 - No.15, September 2015.
[5] A. Osareh, M. Mirmehdi, B. Thomas and R. Markham "Automated identification of diabetic retinal exudates in digital colour images", Ophthalmol, vol. 87, pp. 1220-23, 2003.

[6] Raju Sahebrao Maher, Dnyaneshwar S. Panchal, Sangramsing Kayte, Dr. Mukta Dhopeshwarkar" Automatic Identification of Varies Stages of Diabetic Retinopathy Using Retinal Fundus Images” International Journal of Advanced Research in Computer Science and Software Engineering, Volume 5, Issue 9, September.

[7] National Health Service. The English Diabetic Retinopathy Programme Annual Report, 1 April 200731 March 2008. 2008. [Accessed March 5, 2010]. pp. 89.

[8] Abramoff MD, Suttorp-Schulten MS. Web-based screening for diabetic retinopathy in a primary care population: the EyeCheck project. Telemed J E Health. 2005;11:668-74.

[9] Raju Maher, Sangramsing Kayte, Dnyaneshwar Panchal, Pankaj Sathe, Sandip Meldhe, "A Decision Support System for Automatic Screening of Non-proliferative Diabetic Retinopathy" International Journal of Emerging Research in Management and Technology, Volume4,Issue-10, October-2015.

[10] Cree MJ, Olson JA, McHardy KC, et al. A fully automated comparative microaneurysm digital detection system. Eye (Lond) 1997;11:622-8.

[11] Frame AJ, Undrill PE, Cree MJ, et al. A comparison of computer based classification methods applied to the detection of microaneurysms in ophthalmic fluorescein angiograms. Comput Biol Med. 1998;28:225-38.

[12] Hipwell JH, Strachan F, Olson JA, et al. Automated detection of microaneurysms in digital red-free photographs: a diabetic retinopathy screening tool. Diabet Med. 2000;17:588-94.

[13] Olson JA, Strachan FM, Hipwell JH, et al. A comparative evaluation of digital imaging, retinal photography and optometrist examination in screening for diabetic retinopathy. Diabet Med. 2003;20:528-34.

[14] Spencer T, Olson JA, McHardy KC, et al. An imageprocessing strategy for the segmentation and quantification of microaneurysms in fluorescein angiograms of the ocular fundus. Comput Biomed Res. 1996;29:284-302.

[15] Abràmoff MD, Niemeijer M, Suttorp-Schulten MS, et al. Evaluation of a system for automatic detection of diabetic retinopathy from color fundus photographs in a large population of patients with diabetes.Diabetes Care. 2008;31:193-8.

[16] Niemeijer M, van Ginneken B, Staal J, et al. Automatic detection of red lesions in digital color fundus photographs. IEEE Trans Med Imaging. 2005;24:58492.

[17] Larsen M, Godt J, Larsen N, et al. Automated detection of fundus photographic red lesions in diabetic retinopathy. Invest Ophthalmol Vis Sci. 2003;44:761-6.

[18] Larsen N, Godt J, Grunkin M, et al. Automated detection of diabetic retinopathy in a fundus photographic 
screening population. Invest Ophthalmol Vis Sci. 2003;44:767-71.

[19] Fleming AD, Philip S, Goatman KA, et al. Automated microaneurysm detection using local contrast normalization and local vessel detection. IEEE Trans Med Imaging. 2006;25:1223-32.

[20] Walter T, Klein JC, Massin P, Erginay A. A contribution of image processing to the diagnosis of diabetic retinopathy--detection of exudates in color fundus images of the human retina. IEEE Trans Med Imaging.2002;21:1236-43.

[21] Quellec G, Lamard M, Josselin PM, et al. Optimal wavelet transform for the detection of microaneurysms in retina photographs. IEEE Trans Med Imaging. 2008;27:1230-41.

[22] Karnowski TP, Govindasamy V, Tobin KW, et al. Retina lesion and microaneurysm segmentation using morphological reconstruction methods with ground-truth data. Conf Proc IEEE Eng Med Biol Soc. 2008;2008:5433-6.

[23] Tobin KW, Abramoff MD, Chaum E, et al. Using a patient image archive to diagnose retinopathy. Conf Proc IEEE Eng Med Biol Soc. 2008;2008:5441-4.

[24] Philip S, Fleming AD, Goatman KA, et al. The efficacy of automated "disease/no disease" grading for diabetic retinopathy in a systematic screening programme. $\mathrm{Br} \mathrm{J}$ Ophthalmol. 2007;91:1512-7.
[25] Scotland GS, McNamee P, Philip S, et al. Costeffectiveness of implementing automated grading within the National Screening Programme for diabetic retinopathy in Scotland. Br J Ophthalmol. 2007;91:1518 23.

[26] Niemeijer M, Abramoff M, van Ginneken B. Information fusion for diabetic retinopathy $\mathrm{CAD}$ in digital color fundus photographs. IEEE Trans Med Imaging. 2009;28:775-85.

[27] Niemeijer $M$, van Ginneken B, Cree $M J$, et al. Retinopathy online challenge: automatic detection of microaneurysms in digital color fundus photographs. IEEE Trans Med Imaging. 2010;29:18595

[28] Niemeijer M, van Ginneken B, Russell SR, et al. Automated detection and differentiation of drusen, exudates, and cotton-wool spots in digital color fundus photographs for diabetic retinopathy diagnosis. Invest Ophthalmol Vis Sci. 2007;48:2260-7.

[29] Lin DY, Blumenkranz MS, Brothers RJ, Grosvenor DM The sensitivity and specificity of single-field nonmydriatic monochromatic digital fundus photography with remote image interpretation for diabetic retinopathy screening: a comparison with ophthalmoscopy and standardized mydriatic color photography.Am J Ophthalmol. 2002;134:204-13. 